\title{
Faire le mythe. Histoire, récit et transformation en Amazonie
}

\section{Carlos Fausto}

\section{(2) OpenEdition}

1 Journals

Édition électronique

URL : http://journals.openedition.org/jsa/2749

DOI : $10.4000 /$ jsa. 2749

ISSN : 1957-7842

\section{Éditeur}

Société des américanistes

\section{Édition imprimée}

Date de publication : 1 janvier 2002

Pagination : 69-90

ISSN : 0037-9174

\section{Référence électronique}

Carlos Fausto, «Faire le mythe. Histoire, récit et transformation en Amazonie », Journal de la société des américanistes [En ligne], 88 | 2002, mis en ligne le 05 janvier 2007, consulté le 14 novembre 2019. URL : http://journals.openedition.org/jsa/2749 ; DOI : 10.4000/jsa.2749 


\title{
FAIRE LE MYTHE. HISTOIRE, RÉCIT ET TRANSFORMATION EN AMAZONIE ${ }^{1}$
}

\author{
Carlos FAUSTO *
}

Par l'analyse des différentes versions d'un mythe parakanã (tupi-guarani) sur l'origine des Blancs, cet article cherche à comprendre les conditions de production des variantes d'un mythe, les mécanismes d'incorporation de l'expérience historique et les processus par lesquels les événements sont condensés sous forme narrative. Les différentes versions parakanã sont comparées à d'autres mythes amazoniens sur l'origine des Blancs, en particulier à la célèbre saga d'Aukê, connue de tous les peuples jê du Brésil Central. Enfin, l'auteur examine quelques questions relatives aux concepts de mythe et d'histoire et propose la notion d'" action mythique » qu'il distingue de la notion occidentale de « faire historique ».

Mots Clés : Amazonie, mythe, histoire, récit, transformation.

\section{Making the Myth. History, narrative and transformation in Amazonia}

By means of an analysis of different versions of a Parakanã (Tupi-Guarani) myth on the origin of the Whites, this article aims at comprehending how these versions are produced, how myths incorporate historical experiences, and how events are condensed in a particular narrative structure. The different versions of the Parakanã myth are compared with other Amazonian myths on the origin of the white people, particularly with the story of Aukê, which is known by all Ge-speaking people of Central Brazil. Finally, the author examines some problems related to the notions of myth and history, suggesting the notion of mythic agency in contradistinction with our notion of making history.

KEY wORDS : Amazonia, myth, history, narrative, transformation.

\section{Fazer o mito. História, narrativa e trasformação na Amazônia}

Por meio da análise de versões de um mito parakanã (tupi-guarani) sobre a origem dos brancos, este artigo procura compreender quais são as condições de produção de variantes de um mesmo mito, quais são os mecanismos de incorporação da experiência histórica, e quais são os processos pelos quais os eventos são condensados na forma narrativa. As diferentes versões

* Museu Nacional-PPGAS, Quinta da Boa Vista s/n, São Cristovão, Rıo DE JANEIRo, RJ 20.940-040 Brasil. E-mail : cfausto@alternex.com.br

Journal de la Société des Américanistes, 2002, 88 : p. 69 à 90. Copyright $\odot$ Société des Américanistes. 
parakanã são comparadas com outros mitos amazônicos sobre a origem dos brancos, em particular com a bem conhecida saga de Aukê, difundida entre os povos de língua jê do Brasil Central. Ao final, o autor discute problemas relativos aos conceitos de mito e história, e propõe a noção de agência mítica, diferenciando-a de nossa noção de fazer histórico.

PALAvras Chave : Amazonia, mito, história, narrativa, transformação.

Mais pourquoi [...] marquer une telle réticence vis-à-vis du sujet quand on parle de mythes, c'est-à-dire de récits qui n'ont pu naître sans qu'à un moment quelconque [...] chacun ait été imaginé et narré une première fois par un individu particulier?

C. Lévi-Strauss, L'Homme mu (1971)

La conquête et la colonisation des Amériques furent un événement considérable non seulement par l'étendue des terres conquises ou leurs effets sociodémographiques, mais aussi par la vaste production d'images et d'interprétations qui prit racine dans la pratique, tout en fournissant des directions pour cette mise en pratique. Dans ce champ d'actions et de dispositions, se produisit un chassé-croisé des interprétations des Amérindiens sur les Européens et des Européens sur les Amérindiens qui, avec le temps, prirent corps et se stabilisèrent sous certaines formes.

Dans cet article, je cherche à explorer un aspect de ce processus : celui des traces laissées par l'histoire du Contact dans des récits mythiques. Ce qui m'intéresse ici c'est l'analyse du processus qui permit la mise en récit de certaines expériences historiques sous forme de mythes et en fit des histoires racontées et transmises oralement. Pour aborder cette question, j'analyserai différentes versions d'un même mythe que j'ai enregistrées, entre 1992 et 1995, chez les Parakanã, un peuple tupi-guarani qui vit dans l'interfluve Xingu-Tocantins, dans l'État du Pará, au Brésil. Je chercherai à identifier les mécanismes impliqués dans la production créative de ces variantes et je discuterai de questions relatives aux notions de mythe et d'histoire.

\section{À L'ORIGINE}

La première relation écrite d'un récit indigène dans lequel figurent des Européens, date du milieu du xvı ${ }^{\mathrm{e}}$ siècle. Pendant l'expérience coloniale française dans la baie de Guanabara, André Thevet a recueilli des épisodes de la création de l'humanité qui lui auraient été racontés par le "roi Tupinambá Quoniambec et d'autres vieux Indiens », publiés dans la Cosmographie Universelle en 1575. Ce récit traite des actes des démiurges qui conduisirent à la genèse et aux différenciations successives des êtres qui habitent le cosmos : différenciation de l'humain et du non-humain, de l'Indien et du Blanc, des amis et des ennemis et ainsi de suite. Le mythe inclut les Européens dans cette série pré-existante et les assimile aux démiurges. Selon Thevet ([1575] 1953, p. 41), les Blancs seraient « les successeurs et vrais enfants » du héros culturel qui, du fait de ses constantes métamorphoses, a été brûlé par les ancêtres des Tupinambá. 
L'assimilation, dans ce mythe, des Européens aux démiurges est compatible avec le terme par lequel on les nomme sur la côte brésilienne : caraíba, mot qui désignait les grands chamans tupi-guarani ${ }^{2}$. Ce rapprochement entre conquistadores et chamanisme était surtout fondé sur l'évaluation des implications pratiques et symboliques de la technologie européenne. La profusion d'objets utiles et inutiles que possédaient les Européens était la manifestation publique et visible d'un pouvoir créatif particulier et d'une relation intime avec d'autres sujets du cosmos (que nous appelons « esprits » par la force de l'habitude). Les missionnaires — surtout des Jésuites — avantagés par cette identification, la cultivaient. La prière représentait pour les Tupinambá une sorte de communication chamanique avec un "Grand Esprit ». Anchieta ([1562] 1988, pp. 235-237) raconte que pendant sa mission chez les Tamoio de l'Iperoig, sur la côte de São Paulo, les Indiens venaient lui demander de parler avec Dieu afin de leur assurer une chasse fructueuse et du succès à la guerre. Yves d'Évreux ([1613] 1985, p. 237) rapporte comment Pacamont, un grand sorcier du Maranhão, vint le trouver pour qu'il lui apprenne à parler avec Dieu, puisque tous deux « étaient censés fréquenter les Esprits ».

La violence des Européens, ou même l'intempérance des punitions divines, ne faisaient pas obstacle à cette identification. Le chamanisme tupi-guarani est, en effet, étroitement lié au cannibalisme et à la prédation : les esprits puissants sont, en général, des êtres bestiaux et de féroces mangeurs de viandes crues, tel le jaguar. Ainsi, si leur capacité de violence mettait les conquistadores dans la position d'ennemis, elle ne démentait pas leur vocation pour le chamanisme.

L'équation des Blancs avec le champ chamanique ne s'est pas limitée à la côte atlantique, mais s'est diffusée dans la forêt au fur et à mesure que la colonisation avançait. Les points de vue indigènes sur ce processus se perçoivent dans l'histoire des interactions entre Indiens et Blancs, en particulier dans les mouvements de résistance indigène qui s'approprièrent des images des conquistadores de manière à exprimer l'inversion dans la relation de conquête. Les mythes, à leur tour, nous ouvrent un accès à la compréhension des significations attribuées à cette relation et des différentes façons d'agir sur elle ${ }^{3}$.

Voyons à présent, d'un peu plus près, certains de ces récits, en commençant par les données parakanã.

\section{UN PEU D'HISTOIRE}

Les Parakanã se composent aujourd'hui d'environ 800 personnes qui habitent dans deux réserves indiennes : une dans le bassin du Tocantins, l'autre dans le bassin du Xingu. Quoique, dans la mémoire parakanã, les premiers contacts avec les Blancs remontent à la fin du XIX ${ }^{\mathrm{e}}$ siècle - à l'occasion de la pénétration des ramasseurs de noix dans la région du Tocantins - leurs ancêtres ont probablement dû être affectés, directement ou indirectement, par le processus de colonisation plusieurs siècles auparavant. Les premières incursions d'Européens, surtout de Français, dans cette région, datent, en effet, de la fin du Xvi ${ }^{e}$ siècle et s'intensifièrent au long du siècle suivant, lorsque les Portugais prirent le Maranhão et fondèrent la ville de Belém en 1616.

Cette date marque le début d'une baisse marquante de la population indigène, causée à la fois par les guerres de conquête, les rachats des captifs, les décimations 
missionnaires et les épidémies, en particulier celles causées par la variole. Au milieu du $\mathrm{XVII}^{\mathrm{c}}$ siècle, l'importante population qui avait habité le bassin du Tocantins était déjà annihilée ou réduite en esclavage. Les survivants s'étaient réfugiés dans des zones retirées et sur des cours d'eau plus petits, dans un mouvement qui a marqué toute l'histoire de la conquête de l'Amazonie. Vieira eut l'opportunité de vérifier ce fait, dès sa première incursion dans la forêt amazonienne, en 1653 : « On l'appelle le fleuve du Tocantins à cause d'une nation d'Indiens de ce nom, qui y habitait lorsque les Portugais arrivèrent au Pará ; mais pour celle-ci, de même que pour plusieurs autres, on ne conserve plus aujourd'hui que la mémoire et les nombreuses ruines d'un petit village » (Vieira [1654] 1943, p. 331).

Les Parakanã sont probablement descendants des rescapés de ce processus de conquête et de dépeuplement. Trouvant refuge aux sources des affluents de la rive gauche du Tocantins, ils se sont maintenus isolés pendant très longtemps, au point de ne pas garder le souvenir d'avoir un jour souffert de la violence de l'épée ou de la virulence des épidémies. Donc, du point de vue des Parakanã, les Blancs n'ont été " découverts » qu'à la fin du XIX ${ }^{\mathrm{e}}$ siècle, à l'époque de la génération des grands-parents des personnes les plus âgées d'aujourd'hui ${ }^{4}$.

La « découverte » coïncide avec le développement de la cueillette de la noix du Brésil et de l'extraction du caoutchouc sur la rive gauche du Tocantins, développement qui a favorisé une augmentation de la population locale et la croissance de l'activité commerciale dans les villes de Marabá et Alcobaça (aujourd'hui Tucuruí). Au xx ${ }^{\mathrm{e}}$ siècle, la construction d'une voie de chemin de fer pour relier ces deux villes entraîne la pénétration de la colonisation et donc des contacts plus fréquents entre les Indiens et les autres habitants de la région. Ce mouvement n'a cependant montré son caractère définitif qu'à partir des années soixante, avec les grands projets de l'État brésilien, telles la construction de la route Transamazonienne et celle de l'usine hydroélectrique de Tucuruí. C'est à cette époque que les différents groupes parakanã, se voyant soumis à l'administration de l'État et à la tutelle de la FUNAI entre 1971 et 1984, furent obligés d'abdiquer bonne part de leur autonomie.

Lorsque, quelques années plus tard, j'ai commencé ma recherche sur le terrain, les Blancs apparaissaient dans quatre mythes différents. Deux récits traitaient spécifiquement de leur origine, ils figuraient en outre dans deux histoires à thématique plus ample. La prolifération des récits fait partie de l'effort pour conceptualiser non seulement la différence entre les Indiens et les non-Indiens, mais encore la diversité interne de ces derniers. Selon mes informateurs, les mythes parlent de Blancs différents qui ont pris des directions divergentes et qui peuvent se comporter de manière distincte. Tous ces récits s'étaient cristallisés dès la fin du xIx ${ }^{\mathrm{e}}$ siècle ; ils sont, en effet, connus des deux blocs parakanã - le bloc occidental et le bloc oriental —, qui se sont scindés vers 1890. Cela semble indiquer qu'en dépit de l'isolement dans lequel les Parakanã vivaient à cette époque, l'expérience du Contact avait jadis été intense et variée. La mythologie, cherchant à rendre compte de la variété, présentait déjà les cicatrices de cette histoire.

Dans ce travail, je prendrai en considération un des récits sur la genèse des Blancs, connu sous le titre « Le Rapt des neveux » (Opega-rerahatawera). Je laisserai de côté l'autre récit, "L'Origine de la douleur et des Blancs », que j'ai examiné par ailleurs comme une transformation de la célèbre saga des jumeaux tupi-guarani ${ }^{s}$. Analysons 
donc le premier mythe, que je résume à partir de la version qui m'a été racontée en 1993 par Iatora, un Parakanã occidental.

\section{EN DEVENANT BLANC}

L'histoire commence par des scènes de fornication inusitées : une femme se livre à un commerce sexuel avec des non-humains. Ses frères sont témoins de ces événements.

Tout d'abord elle va forniquer avec une branche d'arbre. Alors :

«-Qu'est allée faire notre sœur ? ont-ils demandé.

Ils partirent.

— Elle est allée forniquer avec une branche d'arbre.

- Va l'arracher.

Il y est allé et l'a arrachée. Elle a cherché, mais en vain :

- Pourquoi m’a-t-on arraché mon mari ? a-t-elle demandé ».

Ensuite elle a commencé à forniquer avec une liane, mais cette dernière a craqué quand ses frères sont partis à sa recherche. Ensuite ce fut au tour d'un cerf. Elle lui apportait de la bouillie. Les frères ont demandé :

«- Pour qui notre sœur emporte-t-elle cette bouillie?

Ils sont allés voir, se sont approchés.

- Pour le cerf, elle verse la bouillie.

Le cerf l'a prise.

- C'est pour le cerf que notre sœur emporte la bouillie.

- Allons le tuer et le manger.

Ils ont donc fait ainsi. Ils l'ont encerclé, l'ont tué et l'ont découpé. De retour au village :

- Nous avons tué un cerf, notre sæur. Il était là-bas, ont-ils dit.

Elle prit la bouillie et sortit.

— Ici ! Ici ! Voilà de la bouillie, c'est pour toi, dit-elle en vain.

Elle vit le lieu où il avait dormi et où il avait été découpé. Elle est revenue et n'a rien dit.

Aussitôt elle vit un tapir et elle lui apporta de la bouillie.

- À qui notre sœur apporte-t-elle cette bouillie ? Allons le tuer et le manger.

Ils y sont allés.

— Iwaoreore, viens prendre ta bouillie ! a-t-elle appelé.

— Kii ! Kii ! Kii !...

Et le tapir est venu.

- C'est un énorme tapir. Je vais le tuer [dit l'un des frères].

Elle a renversé la bouillie et le tapir l'a mangée. Ils restèrent à observer :

- C'est à un grand tapir que notre sœur apporte de la bouillie, mon frère. Allons le tuer et le manger.

Ils l'ont encerclé. Il a couru. Il est tombé. Ils l'ont découpé et l'ont apporté à la maison tout en chantant.

- Nous avons tué un énorme tapir, ma sæur.

- Où l'avez-vous trouvé ?

— Juste là-bas devant. C'est son maître qui l'a tué, ton frère.

— Je n'en mangerai pas ! a-t-elle dit.

- Allons ma sæurr, mange la côte.

Elle est partie et a emporté de la bouillie. Elle a appelé en vain son familier (te'omawa), son mari. 
Alors elle vit le poisson. Lui apporta de la bouillie. Ceci s'est passé juste avant qu'elle ne s'enrage.

- À qui notre sœur apporte-t-elle de la bouillie ? ont demandé les frères.

- Allons regarder, dirent-ils.

Leurs épouses les ont prévenus :

- Votre sœur se disputera avec vous. Lorsque vous avez tué le tapir, elle a dit qu'elle emmènerait vos enfants ! Laissez-la à sa proie-magique (temiahiwa).

Mais ils allèrent voir.

- C'est pour un poisson que notre sœur emporte de la bouillie. Tuons-le.

Ils battirent le poison de pêche et rapportèrent le poisson dans un long panier fait avec la feuille du palmier d'açai. Ils venaient en chantant.

- Nous avons tué un poisson, ma sœur.

- Où était-il ?

— Là-bas ! Notre grosse proie était près de l'eau ».

Dans ce passage initial, une femme célibataire maintient des relations sexuelles avec des végétaux (un tronc d'arbre, une liane) et des animaux (le cerf, le tapir; le poisson). Comme elle n'a que des frères et des belles-sœurs, elle cherche ses " maris » parmi les êtres non-humains. Pour ce faire, elle a recours à ses capacités oniriques. Lorsque le narrateur dit " alors, elle vit le tapir », il faut comprendre qu'elle le voit en rêve et qu'ensuite en état de veille, elle le fait venir pour en faire son partenaire. C'est ce qu'indiquent les termes utilisés pour les animaux : te'omawa (qui veut dire « animal familier ») et temiahiwa (que je traduis par " proie-magique »). Ce sont des catégories qui s'appliquent aux interlocuteurs oniriques des rêveurs. "Ceux qui véritablement rêvent pour de vrai » (opoahiweté-eté-wa'é) interagissent avec les êtres du cosmos et peuvent les convertir en "familiers » avec lesquels ils maintiennent une relation ambivalente de contrôle et de protection. Ces êtres familiers fournissent les chants des rituels et pratiquent les guérisons chamaniques (Fausto 1999).

La prodigieuse activité onirique de la protagoniste indique qu'elle possède une puissance transformatrice et créatrice sans égal, dont les frères tirent profit pour nourrir leur famille. Si elle traite les animaux comme des humains - comme des êtres capables de communication verbale et sexuelle -, ses frères, quant à eux, les voient comme du gibier et les traitent comme tel : ils s'évertuent à objectiver ceux que leur sœur rendait sujets. Ce passage initial du mythe joue en résonance de cette confrontation de perspectives - les maris de la sœur sont le gibier de leurs beaux-frères par la répétition, en parallèle, du même déroulement avec des victimes différentes.

La séquence suivante du mythe offre un dénouement à ce dilemme. La femmechamane, irritée par les attitudes prédatrices de ses frères, rêve de ses neveux $(\mathrm{BCh})$ et les transforme en ses téomawa oniriques. Ainsi, elle les contrôle et les emmène se baigner dans le fleuve. Ils allument un feu sur une pierre et commencent à plonger. Le bain est décrit comme une succession de plongeons et de sorties de l'eau pour que les enfants puissent se réchauffer près du feu. Cette succession froid-chaud rapproche ce bain de ceux revivifiants et transformateurs qui apparaissent, dans la mythologie amérindienne, associés aux thèmes de l'immortalité et du changement de peau. Et, de fait, une transformation se produit peu à peu. Lors des séances qui se répètent pendant plusieurs jours, s'eflectue la séparation entre les futurs Blancs (toriroma) et ceux qui restèrent, les Awaeté ${ }^{6}$.

La séparation est décrite comme un éloignement progressif tout au long du cours de la rivière. Les mères voient encore les plongeons dans l'eau de leurs enfants déjà fort 
loin. Elles les appellent en vain. Rien ne les attire, pas même l'invitation à venir manger du foie de tortue - gourmandise sans égal —, indiquant qu'ils ne partagent déjà plus les mêmes goûts :

« Les mères leur crient :

- Venez manger un bout de foie de tortue.

- Qu'est-ce qu'il y a ? demandent-ils.

- Venez manger un bout de foie de tortue.

- Ils plongèrent.

— Quoi ??? Oh! Mon fils s'est fait complètement Blanc [toria] ! ».

Les enfants sont vus pour la dernière fois sur un rocher, le seuil d'un nouveau monde ${ }^{7}$. Ils se chauffent au soleil. Les parents les appellent, mais ils s'en vont, plongeant dans le fleuve. À partir de là, la séparation devient irréversible. Les appels répétés et frustrés donnent lieu à la violence. Les frères essayent de tuer celle qui a dévoyé leurs enfants, mais elle s'enfuit auprès de ses neveux et ils deviennent tous définitivement des Blancs.

\section{QUI CONTE UN CONTE...}

J'ai enregistré trois versions de ce mythe auprès de trois narrateurs différents. Deux d'entre eux sont des Parakanã occidentaux et l'autre un Parakanã oriental ${ }^{8}$. Ce dernier; en racontant le mythe, n'a même pas fait allusion à la transformation en Blanc. Il a fallu que je lui pose des questions sur le dénouement du récit, que je connaissais déjà, pour qu'il me dise que les neveux sont devenus des Blancs, probablement américains et que, de là, ils sont partis en bateau ${ }^{9}$.

Les deux autres versions, au contraire, annoncent dès le milieu de l'histoire que le destin des enfants est de devenir des Blancs. Toutefois, l'une d'elles, la version d'Iatora, diffère des autres parce qu'elle n'est pas interrompue par la séparation. Elle se poursuit avec un épisode sur l'échec de la visite des parents aux enfants blancs. À partir de là, le mythe prend la forme d'un récit « historique », ou, plus précisément, d'un discours se déployant sur un seul plan existentiel : celui des relations sociales visibles entre les humains. L'univers transformationnel-chamanique de la première partie du récit disparaît dans la deuxième partie.

Ainsi, après avoir constaté que les fils sont devenus des Blancs, les parents décidèrent de partir à leur recherche :

"- Suivons le fleuve pour chercher nos enfants, mon frère.

Ils sont partis : là ils ont dormi, là ils ont dormi, là ils ont dormi. Ils avaient mangé toute la farine. Ils sont revenus vers leurs épouses pour qu'elles en torréfient plus ${ }^{10}$.

- Suivons le grand fleuve à la recherche de nos enfants, mon frère.

Ils partirent, laissant leurs épouses. Ils allèrent jusqu'au pied de la colline, près du fleuve. Ils traversèrent plusieurs montagnes. Ils entendirent alors quelqu'un qui était en train de fabriquer une pirogue.

— Voilà ! Voilà ! ».

Pour les auditeurs parakanã, l'association entre la fabrication de la pirogue et les Blancs est immédiate, puisque, avant le Contact, ils ignoraient cette technologie. Cet 
épisode indique que les parents sont certains d'avoir retrouvé leurs enfants, car la transformation en Blanc est, disons, un résultat anticipé du récit. Les parents continuent leur progression, s'approchant de la pirogue en construction. Cependant, le constructeur s'éloigne pour chercher de la paille du palmier babaçu. À son retour; il s'aperçoit finalement de la présence des Indiens :

« Ils restèrent à regarder la pirogue. Près de l'eau, il [le Blanc] arrachait de la paille. La maison était là. Alors il revint vers la pirogue et repartit tout de suite.

— Qu'a-t-il dû aller chercher en courant ? se demandèrent les pères.

Il courut et prit le fusil.

— Eh ! Qui êtes-vous ? cria-t-il.

Il commença à leur tirer dessus. Les pères s'enfuirent et allèrent retrouver leurs épouses : "Nos enfants ont tiré sur nous!"'".

À ce moment le narrateur se pose une question : « Comment ont-ils fabriqué si vite le fusil ? ». Iatora anticipe une question probable des enfants qui - au côté de leur famille - écoutaient l'histoire qu'il me racontait. La fabrication du fusil, objet qui définit l'identité de ses détenteurs, semble davantage exiger une explication que ne le faisait, dans la première partie du mythe, l'interaction avec les animaux, celle-ci allant de soi.

Cette deuxième partie du récit n'introduit pas une interaction effective avec les Blancs et ne dément pas la première partie, qui nous parle d'une séparation irréversible. Les tirs confirment la transformation ontologique des ex-enfants et réaffirment la conclusion du mythe. Cependant, Iatora ne s'arrête pas là et le thème de la médiation des cadeaux qui a tellement marqué l'expérience de vie du narrateur, suit immédiatement. Après que les épouses se sont réunies et que les pères leur ont raconté ce qui s'était passé, ils décidèrent de prendre la fuite :

«-Allons-nous en, éloignons-nous d'eux. Peut-être suivront-ils le fleuve jusqu'à nous et en finiront-ils avec nous, dirent-ils.

Ils partirent loin dans cette direction et se fixèrent près d'une palmeraie d'açaí.

Quelque temps après :

- Allons chercher des boutures de manioc.

Ils partirent. Ils ont cherché le chemin de l'ancien village. Ils suivirent le chemin. Là-bas se trouvait la maison communale. À l'intérieur, ils trouvèrent des hamacs. Ils firent de la farine avec le manioc qu'ils avaient planté et partirent en emportant les hamacs ".

De nouvelles références historiques apparaissent : les parents ont peur d'être poursuivis et décident d'abandonner le village pour se fixer dans une palmeraie d'açaí (c'est-à-dire qu'ils se réfugient loin du cours du fleuve principal). Pendant ce temps, les enfants-Blancs vont jusqu'à l'ancienne habitation et laissent des hamacs accrochés (un cadeau apprécié puisque les hamacs des Blancs, fabriqués de fils de coton aux mailles serrées, sont beaucoup plus confortables que ceux des Parakanã, faits de fibres de feuille de palmier au tissage plus grossier). Les parents décident de retourner à leur ancien village pour chercher des boutures de manioc, car ils sont partis sans en emporter ; là, ils trouvent les hamacs. Ils font de la farine et prennent les " cadeaux ». Une fois de plus, ils se réunissent avec les femmes et leur montrent ce qu'ils ont apporté : 
«- Regardez ce que nous avons apporté : des hamacs.

Et tout de suite elles répondirent :

- Allons voir nos enfants, allons vivre avec eux, allons devenir des Blancs dans la maison de nos enfants.

Mais ils eurent peur :

- Ils vont en finir avec nous. Ce sont ceux qui portent le vrai arc [le fusil], ils sont devenus complètement des Blancs ".

Ainsi se finit le récit d'Iatora. Il inverse le sens de la séparation de la première partie du mythe : ce ne sont plus les futurs Blancs qui s'éloignent, mais les Indiens qui les fuient. Dans ce parcours, le mythe s'approche chaque fois plus d'un rapport historique. $\mathrm{Si}$, dans la première partie, on raconte la différenciation des Blancs à partir d'une identité originale en clef chamanique (la séparation s'accompagne d'une métamorphose liée à la capacité supra-humaine de la tante paternelle), dans la deuxième partie, la clef est socio-historique. Mais, ici, il est également possible de distinguer deux moments. Initialement, les Awaeté vont à la rencontre des ex-enfants et sont traités comme des ennemis, ce qui les oblige à un éloignement maximum : ils abandonnent le village et partent sans prendre de manioc. Ce fut une expérience commune dans l'histoire de la colonisation de l'Amazonie et les Parakanã occidentaux l'ont vécue, encore que de façon atténuée, au $\mathrm{xx}^{\mathrm{e}}$ siècle, lorsqu'ils ont dû abandonner leurs villages et chercher des régions indemnes de pénétration non indigène. Dans la partie finale du mythe, néanmoins, les Blancs montrent des dispositions pacifiques, laissant des cadeaux dans le village inoccupé que les anciens habitants retournent visiter de temps en temps. Le narrateur attribue alors à la peur des Indiens le fait qu'ils ne soient pas allés vivre avec les Blancs. La conclusion ne dément pas entièrement les autres versions du mythe, en conservant une certaine distance par rapport à aujourd'hui où ils vivent avec les Blancs ; en même temps, elle met l'accent sur un fait qui, diplomatie oblige, peut faire plaisir à l'interlocuteur (lui aussi un étranger distributeur de cadeaux).

\section{LA DIFFÉRENCE QUE FAIT LE CADEAU}

Les cadeaux occupent une position centrale dans l'histoire parakanã du contact. Pendant des dizaines d'années, les Parakanã occidentaux ont fréquenté le Poste de Pacification du Tocantins, fondé en 1928 par le Service de Protection aux Indiens (SPI) pour entrer en contact avec les « sauvages » qui entravaient la construction de la ligne de chemin de fer devant relier Alcobaça à Marabá. Lors de ces visites, ils recevaient des centaines de biens : des haches, des machettes, des couteaux, des hamacs, des moustiquaires, du tabac, de la farine et bien d'autres choses encore.

Les rapports du spi à la fin des années vingt et au début des années trente classaient les Parakanã parmi les « Indiens en voie de pacification » par opposition aux Asurinis, " Indiens guerriers », qui avaient l'habitude d'attaquer la population le long de la voie de chemin de fer. Alípio Ituassu, chargé du poste à l'époque, affirme dans ses rapports que les Parakanã se comportaient en camarades avec les fonctionnaires. La confiance semble avoir été effectivement ressentie par les Indiens qui, lors de cette première période de contacts réguliers entre 1928 et 1938, fréquentèrent le lieu accompagnés des femmes et des enfants, totalisant parfois plus de cent personnes lors d'une même visite. 
Le fait qu'ils emmenaient femmes et enfants - ce qui ne se répétera pas dans la deuxième période des visites, entre 1953 et 1965 - signifie qu'ils ne craignaient ni une agression physique des Blancs, ni une agression chamanique. L'association entre maladie et Blancs prendra corps très graduellement au long du siècle, et encore de façon très timide. Ce n'est qu'après le dépeuplement post-pacification que cette association se consolidera. Les rapports des années trente parlent d'un sentiment opposé à la peur : la joie.

" Le 31 juillet [...], nous avons eu la visite des Indiens qui ont l'habitude de venir dans ce Poste. [...] Après être restés un petit laps de temps, c'est avec la même démonstration de joie qu'ils avaient à l'arrivée, qu'ils se retirèrent : en chantant, en dansant et en bavardant " (SPI 1933).

La démonstration de joie — les chants, le bavardage - allait devenir la marque des visites au Poste de Pacification, ainsiégalement qu'une avidité pour les biens. Beaucoup de cadeaux leur étaient offerts et prenaient une dimension exceptionnelle pour ce groupe qui avait jusqu'alors peu accès à ces objets. Bien que ces grandes fêtes de marchandise aient pu leur faire penser à un renversement de leur destin mythique concernant leur infériorité technique, elles ne les incitaient pas pour autant à envisager la possibilité de devenir Blancs. Comme je l'ai déjà indiqué (Fausto 2001a, pp. 498-504), les fonctionnaires du poste occupaient la même place que d'autres te'omawa oniriques du chamanisme traditionnel parakanã : ils étaient considérés comme des êtres puissants qui étaient néanmoins sous le contrôle des Parakanã, ce qui expliquait d'ailleurs pourquoi ils donnaient tant de choses sans rien exiger en retour. Une des meilleures preuves de l'équation entre ces deux relations, c'est le terme commun appliqué aux Blancs et aux interlocuteurs oniriques : miangá, un vocatif formel pour père et oncle paternel.

Dans les années quarante, lorsque le SPI a commencé à introduire des interprètes indigènes dans le Poste de Pacification du Tocantins (alors rebaptisé Poste d'Attraction Pucuruí), les Parakanã commencèrent à considérer la possibilité de s'unir aux Blancs, voire de devenir totalement Blancs. Iatora, qui était jeune à cette époque, raconte une conversation qu'il aurait eue avec un des interprètes lors d'une visite au Poste un peu plus tard dans les années cinquante :

"Au moment de partir, il [l'interprète] demanda si nous reviendrions, nous avons répondu que oui, nous reviendrions. Il nous demanda combien de nuits nous passerions avant de revenir car ils voulaient nous pacifier. Il demanda si nous n'avions pas peur d'eux. Nous avons répondu que nous n'en avions pas peur :

- Nous sommes frères, nous dit-il.

C'était un Awaeté, un Apyterewa, qui avait été pacifié.

- Ils nous ont pacifiés, nous a-t-il expliqué.

Il n'avait pas abandonné sa langue, c'est la raison pour laquelle il nous parlait comme ça. Il nous a dit qu'au début il n'avait qu'un arc, et qu'ensuite il a reçu un fusil. 11 m'a raconté qu'ils avaient lancé des flèches sur les Blancs :

— Nous les avons fléchés. Alors ils [les Blancs] nous ont dit : les Awaeté vont en finir avec nous. Alors ils nous ont pacifiés.

Nous lui avons demandé :

- Pourquoi avez-vous lancé des flèches sur les Blancs? Tenez-les tout simplement, avons-nous dit à Je'e'yngoa.

Je ne sais pas pourquoi il ne portait pas de short. Bon, les Blancs venaient de l'emmener. Il ne portait qu'une chemise » (Iatora 1993, cassette 37). 
Je'e'yngoa était un Indien tembé ou ka'apor. Il ne parlait pas la langue de nos visiteurs, mais une autre langue tupi-guarani. Toutefois, il était capable de communiquer avec eux bien mieux que les Blancs. Dans ce cas, l'interprète - qui avait la lèvre percée, mais ne portait plus de labret — ressemblait encore à un Awaeté, quoique déjà en voie de transformation. Remarquez l'attention d'Iatora à l'absence du short: Je'e'yngoa s'était habillé comme un Blanc, mais uniquement à moitié. Comme la « pacification » était récente, il n'était pas encore devenu un Blanc : il avait un fusil et une chemise, mais parlait une autre langue et n'utilisait pas de short.

Ce qui intéressait les Parakanã dans l'image de l'Indien récemment contacté, c'était la possibilité d'acquérir les caractéristiques distinctives des Blancs, lesquelles manifestent de façon visible les capacités qu'ils leur attribuent. Comme le suggère Viveiros de Castro, si le corps est un substrat pragmatique et cognitif des opérations d'identification et de différentiation dans les cosmologies amazoniennes, les processus de la métamorphose corporelle sont l'équivalent indigène de notre conversion spirituelle (1996, p. 132). Et cela s'applique à la conversion non seulement des Indiens en non-Indiens, mais aussi d'un non-Awaeté en Awaeté, conversion pensée comme un processus graduel d'adoption de nouvelles marques, de nouvelles dispositions corporelles et d'apprentissage de la langue. Ce processus, mis en acte par la convivialité et par la commensalité, a été éprouvé par les nombreuses femmes étrangères capturées par les Parakanã occidentaux, au $\mathrm{xx}^{\mathrm{e}}$ siècle. Le verbe -mo'yng est employé pour décrire aussi bien le processus d'《 apprivoisement » des Indiens par les Blancs, que celui des femmes étrangères par le groupe. Dans un cas comme dans l'autre, il implique du point de vue indigène une métamorphose corporelle. Dans l'expérience parakanã, cela s'est notamment manifesté par l'abandon, immédiatement après le Contact, de la perforation labiale, puis par l'adoption des vêtements qu'ils avaient jusqu'alors méprisés ${ }^{11}$.

Iatora m'a raconté sa version du mythe du « rapt des neveux » quand ils étaient déjà " pacifiés ». Son récit annonce une réconciliation restée inachevée puisque ses aïeux, dit-il, ont eu peur des Blancs ${ }^{12}$.

\section{Production ET REPRODUCTION DU MYTHE}

Il s'agit maintenant de s'interroger sur le statut de la version d'Iatora, puisqu'elle est la seule, parmi celles que j'ai enregistrées et que j'ai entendues, à incorporer ces événements finaux au récit. Indique-t-elle une transformation en cours du mythe ou s'agit-il d'une simple invention individuelle? Afin d'éclaircir ce point, je ferai quelques observations sur la personnalité d'Iatora et surtout sur le contexte dans lequel les mythes sont racontés chez les Parakanã occidentaux.

Contrairement à ce qui se passe chez les Parakanã orientaux, où les mythes sont racontés de préférence au cours de réunions nocturnes masculines, chez les Parakanã occidentaux le récit se fait à l'intérieur des maisons, en présence de la famille du narrateur et s'adresse aux générations plus jeunes. Chaque fois qu'Iatora me racontait une histoire, l'auditoire, formé de garçons et de filles prépubères, était captivé. Ce jeune public, avec ses questions et ses interruptions plaisantes, stimulait Iatora à continuer. Pioma, son épouse, participait de manière active, en anticipant les dialogues, en entremêlant les faits qu'elle avait entendus de son père ou en s'informant sur 
des points obscurs. Dans chacune des situations d'énonciation, le mythe s'actualisait sans grandes contraintes sociales sur sa présentation verbale. Cette situation ouvre un espace aux interprétations personnelles, en particulier chez des individus poussés par leur talent de conteur et par leur imagination fertile, comme c'est le cas d'Iatora, le plus âgé des Parakanã et mon plus cher informateur. Dès qu'il perçut mon intérêt pour ses histoires (et à mesure que je progressais dans la compréhension de la langue), il les rendit chaque fois plus longues et plus détaillées, prenant plaisir à raconter, plaisir qui n'était pas uniquement lié à la perspective de recevoir des cadeaux ${ }^{13}$.

D'ailleurs, les Parakanã occidentaux ne reconnaissent que faiblement des sources socialement légitimes d'énonciation, c'est-à-dire, des persomnes censées posséder une relation privilégiée avec "la tradition ». Bien sûr, l'âge, le sexe et la personnalité sont des éléments importants pour définir qui sont les bons conteurs, mais cela n'implique pas qu'ils soient les interprètes par excellence d'une tradition ancestrale. La culture parakanã, en particulier sa variante occidentale, met plus l'accent sur la production continuelle du nouveau que sur la transmission du même. Les chants, par exemple, une fois exécutés dans le rituel, ne peuvent pas être réutilisés à des fins cérémonielles ou thérapeutiques; il faut toujours produire des chants nouveaux. Dans un tel contexte, ce qui est transmis c'est une matrice inconsciente à partir de laquelle on produit des variantes plutôt que des contenus stables. De plus, ces chants sont censés être le résultat d'interactions oniriques avec des ennemis (Fausto 2001a) et certains rêves donnent lieu à de véritables récits mythiques individuels qui peuvent incorporer des mythèmes très connus. Iatora est un maître de cet art onirique-narratif qui se situe à mi-chemin entre la fabulation individuelle et le mythe collectif.

On voit ainsi qu'il y a non seulement un faible contrôle social sur la variation, mais aussi une valorisation de la capacité innovatrice. Chez les Parakanã orientaux, la situation est différente : ils reconnaissent, en effet, un espace public d'énonciation, la place où la parole des hommes âgés, et surtout celle des chefs, est soumise à l'écoute critique de la collectivité masculine. Mais, dans le même temps, cette parole gagne en légitimité du seul fait d'être proférée dans cet espace. Chez les Parakanã occidentaux, la diffusion des récits et des nouvelles se fait plutôt à travers un système capillaire, domestique et difficilement repérable par l'ethnologue.

De quelle façon, alors, les innovations sont-elles collectivement acceptées et reproduites? Comment, au fil du temps, de nouvelles versions du mythe acquièrent-elles une forme plus ou moins stable? Voilà des questions pour lesquelles je n'ai pas de réponse empirique, du moins pour l'instant. Il serait intéressant de recueillir aujourd'hui d'autres versions de l'histoire du rapt des neveux, pour savoir si l'interprétation inventive d'Iatora s'est déjà cristallisée dans une variante en circulation. Quand bien même serait-elle restée sans lendemain que j'aimerais néanmoins suggérer, au moyen d'une comparaison, qu'elle décrit bien les mécanismes d'élargissement du mythe, tout comme elle nous permet d'entrevoir les processus de condensation par lesquels la forme mythique intègre l'expérience historique.

\section{MYTHOGRAPHIE}

À des fins de comparaison, je vais prendre l'un des plus célèbres mythes sudaméricains sur la genèse du Blanc dans la littérature ethnologique. Je me réfère à 
l'ensemble des histoires connues sous le nom timbira de son protagoniste, Aukê, lequel apparaît sous d'autres désignations chez les Apinajé (Nimuendajú 1956) et chez certains groupes kayapó (Vidal 1977 ; Turner 1988a). Voici un résumé de la version canela recueillie par Nimuendajú (1946, pp. 245-246) :

" On raconte qu'une femme a entendu le cri d'une sarigue [préa] pendant qu'elle se baignait dans le fleuve. Arrivée à la maison elle s'aperçut qu'elle était enceinte car l'enfant-sarigue lui a parlé de l'intérieur de son ventre, lui annonçant le moment de sa future naissance. La mère l'a alors averti : "Si tu es un garçon je vais te tuer, mais si tu es une fille je vais t'élever ". Aukê était un garçon et, à sa naissance, sa mère l'a enterré. La grand-mère, cependant, l'a retiré de la tombe et l'a allaité. Aukê a grandi rapidement et tout de suite s'est montré capable de se transformer en différents animaux. À cause de ces transformations, son oncle maternel a essayé à plusieurs reprises de le tuer, mais il ressuscitait toujours. À la fin, l'oncle a assommé l'enfant et l'a incinéré. Ensuite tout le monde est parti. Passé un certain temps, la mère a demandé qu'on lui apporte les cendres d'Aukê et deux hommes sont retournés sur le lieu de l'assassinat. Ils découvrirent alors qu'Aukê était devenu un Blanc et qu'il avait créé les Noirs, les chevaux et le bétail avec du bois. Aukê les reçut gentiment et invita sa mère à venir habiter chez lui. »

J'aimerais suggérer que les versions d'Aukê et celle d'Iatora contiennent plusieurs strates qui représentent différents moments de réflexion et de condensation mythiques. Lorsque j'emploie le mot condensation, je me réfère à un processus de réduction (au sens photographique) du récit, qui tend à accentuer les discontinuités synchroniques au détriment des continuités diachroniques, à permettre de nouveaux arrangements des unités à travers l'élision ou l'inclusion de motifs, enfin à utiliser des mécanismes formels (poétiques, au sens de Jakobson) tels que le parallélisme.

La première strate ne dépend pas de la présence du Blanc. Dans les deux cas, il est possible de raconter la même histoire sans inclure cette métamorphose. Le mythe parakanã pourrait s'arrêter avec le départ des neveux emmenés par la tante paternelle ${ }^{14}$. Il pourrait être compris alors comme une inversion de l'échange matrimonial parakanã, dans lequel ce sont les frères qui prennent les filles de leurs sœurs pour eux (mariage avunculaire) ou pour leur fils (mariage entre cousins croisés). Dans le mythe, au contraire, les hommes tuent leurs "beaux-frères ", contraignant leur sœur au célibat. Celle-ci, au lieu de leur donner des épouses, leur vole leurs fils. Le message est clair : il vaut mieux incorporer des beaux-frères (aussi étrangers qu'ils puissent être) que maintenir des sœurs dans le célibat. Comme nous l'avons vu, la version que j'ai recueillie chez les Parakanã orientaux s'interrompt à ce moment précis de l'histoire. Je suggère qu'il doit être également possible d'interpréter le mythe d'Aukê sans faire référence à l'irruption des Blancs.

Le deuxième moment de condensation est l'incorporation des Blancs au récit, lorsque l'on constate la métamorphose de ceux qui se sont séparés ou qui ont été abandonnés. Le mythe explique l'apparition du Blanc, lui donne une place dans le système de classification indigène, sans rien dire sur le contenu des relations entre les Indiens et les non-Indiens; il l'insinue, toutefois, lorsqu'il associe les Blancs aux capacités chamaniques ${ }^{15}$. La version racontée par Pi'awa correspond à ce niveau de condensation : avant la fin de l'histoire, on sait déjà que les garçons se transformeront en Blancs (les épouses disent à leurs maris : « Notre belle-sœur a emmené nos fils en les transformant en Blancs »). La genèse du Blanc est un résultat anticipé déjà contenu dans la structure du mythe. C'est là le niveau de condensation atteint par les Parakanã 
occidentaux, chez qui l'histoire peut aussi être référée comme Toriroma (« les Futurs Blancs »).

La troisième strate des récits concerne une réflexion sur le mode d'interaction entre Indiens et non-Indiens, conservant une série de références qui, de notre point de vue, peuvent être reconnues comme « historiques ». Dans les mythes jê, cette strate correspond à la description finale de la rencontre avec le protagoniste qui porte déjà sa nouvelle identité. Je résume maintenant la version apinajé, recueillie également par Nimuendajú (1956, p. 127) :

«Tous croyaient que Vanmegaprána [Aukê] était mort. Néanmoins il avait ressuscité de ses cendres en tant que Blanc. Il était allé au bord de la rivière et avait lancé de la farine de manioc aux poissons : immédiatement les poissons blancs s’étaient transformés en gens blancs et les poissons noirs en Noirs. Puis il a fait une grande maison et toutes les choses que les chrétiens possèdent aujourd'hui. À l'aube, les Indiens du village ont entendu le chant du coq très loin de là ; puis ils ont entendu les voix des chevaux et des vaches. Après, ils ont vu la fumée au loin et ils ont constaté qu'ils avaient des voisins. L'un d'eux décida d'aller jusque là-bas et Vanmegaprána lui a montré les animaux domestiques et lui a dit leur nom. Après, il a appelé les gens de sa famille ; il leur a donné du riz et de la viande de bœuf à manger et leur a enseigné comment devaient être préparés ces aliments. À son oncle il a dit : " si tu ne m'avais pas poursuivi, tu serais maintenant un homme riche ". Après il a demandé à Nyimògo [sa mère] si elle le reconnaissait. Elle lui a répondu que non, alors il lui a dit qu'il était son fils. Nyimoogo a beaucoup pleuré. Vanmegaprána a donné plein de cadeaux à sa famille et l'a renvoyée en paix. Vanmegaprána était le vieil empereur Don Pedro II ».

Le récit explique en clef chamanique comment Aukê s'est multiplié (en transformant les poissons), mais, à partir de là, il prend un ton " historique » qui offre des similitudes avec les épisodes finaux du mythe parakanã conté par Iatora. Comme le fait remarquer DaMatta (1970, p. 99), le mythe d'Aukê ne se déroule pas dans un temps proprement mythique, mais met en relation des périodes de temps discontinues. Selon cet auteur, la dernière partie est justement celle qui permet une plus grande liberté narrative et qui présente une plus grande divergence entre les diverses variantes, indiquant par là un niveau de condensation du récit, disons, moins profond. Contrairement à l'innovation d'Tatora, l'histoire s'est, de toute façon, établie et généralisée parmi les groupes timbira et kayapó. La référence à Don Pedro II fait, en outre, supposer que le récit a pris cette forme stable pendant la seconde moitié du XIX ${ }^{\mathrm{c}}$ siècle, lorsque l'expansion de la frontière économique sur les territoires timbira du Maranhão et de Goiás était déjà devenue irréversible ${ }^{16}$.

En comparant le mythe d'Aukê avec la cosmogonie tupinambá recueillie par Thevet au $\mathrm{XVI}^{\mathrm{e}}$ siècle, Lévi-Strauss $(1991$, p. 82) remarque que le mythe jê adopte une solution "moins radicale » que le second (dans lequel la disjonction Indiens/ non-Indiens est irréversible), car elle suggère la possibilité d'une conversion tardive des Indiens en Blancs, pour qu'ils puissent profiter de leurs « trésors ». Selon l'auteur, la raison de cette différence se trouve dans la diversité des expériences de contact : les Timbira s'interrogeaient sur notre société dans une situation bien plus défavorable que celle des Tupis du XVI ${ }^{\mathfrak{e}}$ siècle, époque où les envahisseurs étaient une faible minorité. En revanche, les Timbira étaient obligés d'accepter les Blancs, si bien que le mythe suggère une réconciliation : Aukê offre des " cadeaux » aux Indiens. 
On retrouve cette distinction entre le mythe jê du XIX ${ }^{\mathrm{e}}$ siècle et le mythe tupi du $\mathrm{XVI}^{\mathrm{e}}$ siècle en comparant les différentes versions du mythe parakanã du « Rapt des neveux ». Alors que la plupart des versions finissent sur un simple constat de séparation, celle d'Iatora va plus loin : tout d'abord, elle donne un contenu à la disjonction (les Blancs possèdent des fusils, les Indiens n'en possèdent pas) ; ensuite, elle propose une possibilité de résorber cette disjonction (les Blancs laissent des cadeaux pour les Indiens). La version d'Iatora apparaît comme une possibilité d'interprétation, à partir du mythe, de l'expérience du Contact et, en même temps, comme une possibilité de réinterprétation du mythe à partir de cette expérience. Iatora cherche non seulement à donner du sens à l'histoire au moyen du récit, mais aussi à actualiser la signification du récit au moyen de l'histoire. Le fait que ce mouvement de double sens se cristallise en une forme plus ou moins stable reste néanmoins une question ouverte.

\section{L'ACTION MYTHIQUE}

J'ai montré que les cicatrices des expériences les plus reculées du Contact se manifestent par la multiplication des mythèmes sur l'origine des Blancs et que l'innovation d'Iatora exprime une réflexion autochtone sur une expérience historique plus récente. Le narrateur accueille les nouveaux faits et articule le temps du mythe sur celui de l'histoire, respectant néanmoins l'écart nécessaire entre le temps du récit et le temps de son actualisation verbale. Cet acte d'élargissement du mythe produit à son tour le mouvement que j'ai appelé condensation mythique, procédé par lequel l'expérience historique devient mythologie, s'éloignant non seulement de la matérialité de l'action (ce qui serait propre à tout récit), mais aussi des autres genres narratifs que l'on aurait tendance à identifier comme « historiques » (et à utiliser comme « histoire orale »). Fréquemment, outre les aspects formels déjà cités, la condensation mythique semble impliquer une «chamanisation » croissante du récit, faisant intervenir des capacités extraordinaires pour expliquer les événements, en particulier ceux qui contiennent des processus de transformation.

Il est néanmoins important de remarquer que les Parakanã ne font pas la distinction entre deux genres de récits, l'un qui correspondrait au «mythe », l'autre à «l'histoire». Bien qu'il y ait une catégorie qui s'applique mieux au premier - morongeta-imyna, « conversation ancienne » - , la distinction ne se fait pas sur le plan d'une classification extérieure, mais plutôt sur les indices intérieurs au récit. Le mythe est une histoire véritable dont l'origine de l'énonciation est méconnue. Comme l'a remarqué Ireland (1988, p. 163) pour les Waurá, la distinction entre récits « mythiques » et récits « historiques » correspond à la distinction qui peut se faire entre des faits si anciens qu'il est impossible d'identifier ceux qui en ont été témoins et des faits dont on peut préciser la chaîne des témoins (voir également Gallois 1994, pp. 21-26). Dans ces cosmologies où le statut des récits et de toute information dépend du crédit porté au témoignage de celui qui les a vécus et de celui qui les a racontés, les mythes apparaissent comme l'unique histoire véritable puisqu'elle ne dépend pas de la source d'énonciation ${ }^{17}$. Les mythes n'expriment pas une perspective particulière, car ils s'appliquent à la compréhension elle-même des diverses perspectives contenues aujourd'hui dans le monde. 
En revanche, le critère que nous utilisons afin de distinguer entre le mythe et l'histoire serait plutôt la capacité d'agir sur le monde attribuée ou non aux protagonistes du récit. Là où on reconnaît la capacité d'action des humains dans leurs attributs ordinaires, on identifie un récit historique. Hérodote (1952) débute l'Histoire en affirmant que son objectif est de préserver, au moyen de la parole écrite, ce qui doit son existence aux hommes, de donner de la pérennité aux choses humaines, autrement vouées à la mort et à l'oubli. C'est ce lien entre l'action humaine et la mise en récit que nous cherchons à identifier dans ce que nous appelons l'histoire orale. Elle donne au récit un statut spécial et nous permet de construire, ensuite, notre récit sur l'histoire d'un peuple sans écriture. Le fait que le narrateur ait été le témoin direct de l'événement raconté fonctionne comme une preuve supplémentaire, car nous reconnaissons comme historique tout récit se rapportant à des actions humaines (et nous pouvons l'épurer, par exemple, de ses affabulations, comme nous le faisons fréquemment avec les textes des chroniqueurs).

Ce procédé a son utilité, et je l'ai moi-même utilisé pour reconstruire un siècle d'histoire parakanã (Fausto 2001a). Il comporte, cependant, des risques, non dans ses méthodes, mais dans les implications que l'on a coutume d'en tirer. L'existence de récits que nous qualifions d'historiques devient argument et preuve du nonemprisonnement de la conscience autochtone par la « machine du mythe ». L'analyse de tels récits apparaît dès lors comme le moyen de dénoncer l'opération occidentale de " primitivisation » de l'Autre et de conférer à cet autre une conscience historique, instaurant capacité d'action (agency) là où il n'y avait que structure. La diffículté que je perçois ici concerne la théorie de l'action. Tout se passe comme s'il n'y avait pas de capacité d'action tant que la praxis humaine n'est pas reconnue comme condition, en soi et pour soi, de la transformation sociale. Je pense au contraire que c'est à nous qu'il revient de se poser justement la question sur la signification de l'" agir » et du « faire » dans des configurations socioculturelles où l'action transformatrice ne se réduit pas à la praxis humaine.

S'il nous semble exister une continuité entre le concept moderne de l'histoire et l'ancien (hellénique classique), en dépit de la différence entre la notion de processus général et celle de fait et de grandeur singuliers, c'est parce qu'aux yeux de la modernité, tous deux s'enracinent dans la pratique humaine ou bien, comme le disait Châtelet, dans la reconnaissance de la "nature sensible-profane de l'existence humaine » (1962, p. 40). La capacité humaine d'action définit la sphère propre à l'histoire. Ce « faire », qui s'applique aussi bien à la société qu'à la nature et qui peut être raconté a posteriori, est une puissance qui ne se réalise comme conscience historique que lorsqu'elle se sait action humaine ; c'est-à-dire que lorsqu'elle se reconnaît comme action créatrice capable de produire des transformations dans le monde social. Cette conscience à la fois historique et politique suppose en outre l'homogénéité de l'avant, du maintenant, de l'après. C'est l'uniformitarisme de Lyell appliqué aux choses humaines ${ }^{18}$.

Certains ethnologues américanistes partagent cette notion de capacité d'action (agency), l'enracinant dans un monde historique pour la rendre incompatible avec l'univers du mythe. Terence Turner, dans les conclusions du livre Rethinking History and $M y t h$, définit la conscience historique comme la perception que le monde social est le produit de l'action créatrice humaine. Dans le «mode mythique de la cons- 
cience ", au contraire, " le pouvoir de créer ou de changer les formes et les contenus de l'existence sociale - l'action sociale dans son sens plein — n'est pas vu comme étant disponible pour les gens du monde social contemporain » (Turner 1988b, p. 244). Pour sa part, Jonathan Hill suggère, dans l'introduction à ce même ouvrage, que la conscience historique implique « une reconnaissance réflexive des acteurs en leur habileté à produire des ajustements situationnels plus durables dans les ordonnances sociales [...] ", reconnaissance qui serait fondée sur le fait que "le passé historique est vu comme habité par des êtres complètement humains et culturels, qui [...] possédaient les mêmes pouvoirs pour produire des transformations que les gens d'aujourd'hui » (Hill 1988, p. 7).

Je n'ai pas l'intention de m'aventurer ici sur le terrain mouvant des notions de conscience historique ou mythique. Ce qui m'intéresse, c'est de remettre en question l'assimilation de la notion de capacité d'action (agency) à un « faire » transformateur qui est vu comme la capacité des êtres humains (en tant qu'humains) à produire des changements dans le monde conventionnel des " ordonnances sociales ». J'aimerais proposer l'inverse : à savoir que les notions de fabrication et de transformation dans les pratiques de connaissance indigènes impliquent le caractère non arbitraire de l'ordre du monde et supposent des relations avec des êtres qui ne sont pas complètement humains. Autrement dit, je suggère, dans ce contexte, que, d'une part, les concepts d'action et de capacité d'action soient liés au problème de la production de transformations dans un monde social qui n'est pas vu comme étant conventionnel et arbitraire et que, d'autre part, l'action transformatrice dépende de l'interaction avec des êtres pouvant être sujets sans être proprement humains.

L'équivalent indigène de ce que nous appelons "capacité d'action historique » (historical agency) serait ainsi l'action chamanique sur le monde. Les divers mouvements de résistance indigène post-Conquête, connus dans la littérature comme millénaristes ou messianiques, en sont un bon exemple ${ }^{19}$. De notre point de vue, ils apparaissent comme des mouvements religieux syncrétiques, irrationnels et réactifs, fruit de l'absence d'une véritable conscience historique capable d'engendrer une action transformatrice sur le monde social. Du point de vue indigène, au contraire, ils apparaissent comme une chamanisation de la pratique, comme une mobilisation de capacités créatrices qui ne sont pas exclusivement "sociales » et qui ne sont pas disponibles de façon "naturelle » aux humains. C'est parce que les humains ne sont pas uniquement humains et que des non-humains partagent des qualités humaines qu'il est possible d'avoir une action créatrice.

La capacité d'action indigène suppose donc la possibilité de produire des transformations dans l'ordre donné par le mythe, et non pas de substituer une convention par une autre convention, un contrat par un autre contrat. L'action transformatrice sur le monde est un acte différenciateur (Wagner 1981) en relation avec l'ordre post-mythique ; elle requiert donc l'actualisation du temps mythique pour effectuer des transformations effectives. Et si le mythe est, comme le veut Turner; " la 'fétichisation' du processus de production de la société » (1998b, p. 243), la forme du faire mythique n'est pas celle de l'action sociale qui se sait exclusivement humaine. L'action créatrice dépend de la mobilisation de capacités qui ne sont pas exclusivement humaines, capacités dont les humains ont été (partiellement) privés dans l'ordre post-mythique. Faire l'histoire est donc une mytho-praxis mise en récit comme passé 
et comme futur en clef chamanique. Ainsi, si l'incorporation des événements historiques aux mythes peut se faire en clef socio-historique, comme cela se passe dans la version d'Tatora, le mythe en tant que discours de transformation - c'est-à-dire comme histoire du futur et source d'action — doit cependant avoir d'abord recours au chamanisme comme condition de possibilité pour produire des changements.

Un mythe des Arapaço du bassin du Uaupés, dans la version recueillie par Janet Chernela, peut nous aider à comprendre ce point en particulier. L'Indien Crispiano Carvalho lui a fait le récit suivant, qu'elle divise en trois parties. Dans la première, connue par tous les autres peuples de la région, les Blancs ne sont pas cités. Dans la deuxième partie, on raconte que l'ancêtre Unurato, mi-serpent, mi-homme, a descendu le Rio Negro et est entré dans l'Amazone. Une nuit, arrivé à Manaus, il a commencé à fréquenter les bars en tant qu'homme. Il dansait, buvait, mais retournait toujours s'immerger dans le fleuve sous sa forme de serpent. Un jour, il demanda à un Blanc de venir le rencontrer à minuit sur la plage. Le Blanc portait de l'eau de vie, un fusil et un œuf de poule ; il devait lancer l'œuf sur Unurato, mais lorsqu'il le vit surgir de l'eau sous forme de serpent, il lui tira dessus avec son fusil. Sous la décharge des plombs, sa peau de serpent tomba dans l'eau tandis que son corps humain resta sur la plage. Le tir ayant détruit ses capacités surnaturelles, il devint alors un homme commun, aveugle d'un œil et il commença à vivre comme n'importe quel autre homme. Crispiano conclut « nous sommes ses descendants. C'est la raison pour laquelle nous sommes appelés Pino Masa, Peuple du Serpent » (Chernela 1988, p. 43).

Le récit ne se termine toutefois pas là. Dans la troisième et dernière partie, Unurato n'est plus un homme commun, il redevient serpent et protagoniste d'une nouvelle histoire :

« Unurato est allé à Brasília et là-bas il a travaillé à la construction de grands édifices. Il a connu toutes sortes de choses - maisons, meubles, taxis — des choses que nous n'avons pas ici [...]. L'année dernière, les eaux ont beaucoup monté. C'était Unurato qui revenait. Il a nagé vers l'amont. Il était un sous-marin géant, mais comme c'est un serpent surnaturel, il a remonté les chutes d'eau. Le sous-marin est ici [...], il apparaît à minuit. Il y a tellement de choses dedans qu'il est impossible de compter le nombre de boîtes dans ce bateau. Il a un éclairage électrique. Avec les machines, les êtres-serpents [wai masa] sont en train de construire une énorme ville sur le fleuve. On peut entendre le bruit de ces machines dans l'eau quand on s'approche de là-bas. Toutes sortes de wai masa travaillent dans ce bateau. Maintenant nous sommes peu nombreux, mais il va nous rapporter la prospérité et la multitude. »(Chernela 1988, p. 43). *

* Manuscrit reçu en janvier 2002, accepté pour publication en février 2002.

\section{NOTES}

1. Une première version de ce texte fut présentée lors du colloque « Tempos indios : histórias e narrativas do Novo Mundo ", organisé par John Manuel Monteiro et moi-même au Museu Nacional de Etnologia (Lisboa). Elle sera publiée en portugais dans les annales du colloque. D'autres versions furent présentées à l'Universidade de São Paulo et à l’École pratique des hautes études (Paris), pendant un séjour en France financé par une bourse de la Coordenaçào de Aperfeiçoamento de Pessoal de Ensino Superior (CAPES). Pour leurs invitations et commentaires, je voudrais remercier Joaquim Pais de Brito, Dominique Gallois, Denise Fajardo et Patrick Menget. Je suis particulièrement reconnaissant à France-Marie Renard-Casevitz et à Philippe Erikson pour leurs observations et corrections détaillées. Je reste toutefois le seul responsable de mes 
prises de position. La recherche chez les Parakanà a été financée par la Financiadora de Estudos e Projetos (FINEP), l'Associação Nacional de Pós-Graduação em Ciências Sociais (ANPOCS), The Ford Foundation, l'Universidade Federal do Rio de Janeiro (UFR) et The Wenner-Gren Foundation for Anthropological Research. La première version de ce texte a été traduite par Christine Guimaràes.

2. Au xvi ${ }^{e}$ siècle, il y avait des distinctions par nationalité : les Français étaient appelés Mair (comme le démiurge) et les Portugais Peró. Encore aujourd'hui, divers groupes indigènes appellent les Blancs caraiba ou caraí. Quant aux Parakanã, ils utilisent un terme restreint aux peuples du système Tocantins-Araguaia, toria, et réservent le mot karahiwa pour désigner les chants thérapeutiques.

3. Il y a une remarquable récurrence des quelques thèmes mythiques qui se sont annexé le Blanc; on les trouve aujourd'hui disséminés chez des peuples de régions distantes et de familles linguistiques différentes. Ces thèmes parlent de la séparation entre les hommes et les démiurges, de l'instauration du régime de l'existence humaine, de la mort et du travail. Les Blancs y apparaissent comme sujets à une existence de moindre privation : moins de travail (parce que, lorsque le démiurge a présenté les objets culturels indigènes et les armes et les outils de métal, l'ancêtre des Indiens a choisi les premiers alors que le futur Blanc a préféré les autres), et à une vie plus longue (parce que les Blancs connaîtraient les secrets chamaniques de la revivification, en particulier le changement de peau, fréquemment associé au changement vestimentaire). Voir Fausto 2002.

4. Les Blancs eux aussi « découvrent » les Indiens durant cette même période. Le manque de continuité de la conquête de l'Amazonie a permis une série de re-découvertes : quelques-uns des peuples considérés comme non contactés par des anthropologues, des missionnaires et des agents de l'État au xx ${ }^{\mathrm{e}}$ siècle avaient été sous l'influence directe du système colonial plusieurs siècles auparavant. Voir Fausto $2001 \mathrm{~b}$.

5. Dans cette analyse, je cherche à indiquer comment le Blanc apparaît associé à la puissance rénovatrice du chamanisme et, en particulier, à la capacité de franchir les limites entre la vie et la mort - associations qui, comme nous l'avons vu, surgissent dès le premier siècle de la conquête en Amérique du Sud. Voir Fausto 2001 a, pp. $470-482$.

6. Awaeté peut être traduit par « vrais humains » ou " humains par excellence ». Il ne s'agit pas d'une auto-désignation, mais d'une catégorie à « extension variable » qui s'applique a minima à ceux qui partagent la même langue, les mêmes coutumes et les mêmes marques corporelles.

7. À propos du rôle de semi-conducteur des rochers dans l'eau, voir Lévi-Strauss (1991 p. 69 ; 1971 p. 388 ; pp. $398-400)$.

8. La version enregistrée chez les Parakanà orientaux a été racontée par Pykawa, aujourd'hui chef du village de Paranowa'ona. La troisième version provient de Pi'awa, un Parakanã occidental comme Iatora ; il habite le village de Maroxewara où on le considère comme un excellent narrateur.

9. Dans les mythes d'autres peuples d'Amazonic, ce déplacement en aval explique également la séparation-origine des Blancs. Le mouvement dans le sens inverse (en amont) peut aussi servir à expliquer le retour des Blancs (ou de leurs marchandises) : par exemple, dans le mythe wayãpi d'Ulukauli rapporté par F. Grenand (1982, pp. 241-285) et dans ceux de peuples du haut Rio Negro.

10. La fin des réserves de farine de manioc sert d'indice pour évaluer la distance à parcourir pour rejoindre leurs enfants-devenus-Blancs.

11. Pendant leurs visites au Posto de Pacificação do Tocantins dans les années trente, les Parakanã recevaient des vêtements des fonctionnaires du SPI, mais, en partant, ils les abandonnaient aux alentours du Posto.

12. Contrairement à ce que lui raconta Je'e'yngoa, pour qui les Blancs avaient pacifié les Indiens parce que, eux, en avaient eu peur.

13. Je distribuais de manière équitable ces objets quand j'arrivais. Toutefois, lors de mon départ, je laissais toujours un certain nombre d'objets m'appartenant, en particulier pour Iatora. Jamais, néanmoins, il n'y eut un échange direct entre parole et cadeaux. J'ai toujours gardé l'illusion (confortable pour l'ethnographe solitaire) qu'il y avait un lien affectif spécial qui nous liait, illusion manipulée par de jeunes Parakanã, qui me téléphonaient d'Altamira en me disant que je devais leur rendre visite, car mon " oncle paternel » se languissait beaucoup de moi.

14. Il pourrait aussi finir, comme me l'a suggéré F.-M. Renard-Casevitz, par la transformation des neveux en poissons ou en une entité surnaturelle. En fait, il existe un mythe très semblable à celui-ci raconté par les Asurini du Xingu, dans lequel la sour, après avoir vu ses « maris " (le cerf et le tapir) tués par ses frères, remonte le fleuve et part habiter là où « l'eau rencontre le ciel », se transformant en un être surnaturel (Müller 1990, pp. 336-337).

15. Il y a des mythes qui déterminent clairement le mode d'interaction entre les Indiens et les non-Indiens. Tous ceux qui font usage du fameux motif du « mauvais choix » (voir note 3 ) ont cette caractéristique. 
16. L'élevage s'est diffusé à partir de la moitié du XvıII ${ }^{e}$ siècle grâce à la découverte de prairies naturelles dans le Maranhão. De là, il s'est répandu en direction du Tocantins et de Goiás, donnant naissance à de nouvelles bourgades et à de nouveaux villages. C'est un moment de pression sur les territoires des Timbira, qui furent progressivement encerclés par le front pionnier, jouant, par moments, le rôle d'alliés éventuels pour les guerres de conquête, à d'autres, celui de victimes de ces mêmes mouvements (Hemming 1987, pp. 181199 ; Melatti 1967, pp. 32-43). Les actions guerrières, tout comme les trêves, s'accompagnaient d'épidémies provoquant un fort dépeuplement tout au long du siècle. Les Apinajé, par exemple, en contact permanent avec la société nationale depuis 1797, ont été ravagés par la variole en 1817 et sont entrés en conflit avec d'autres Indiens et des non-Indiens dès les premières années du xix ${ }^{e}$ siècle. Malgré tout, il en restait plus de quatre mille dans les années 1820. En 1859, ils n'étaient plus que deux mille et, en 1926, ils étaient à peine cent cinquante (Nimuendajú 1956, pp. 4-6).

17. Dans maintes langues indigènes d'Amazonie on trouve des marques, des termes et des formules " citatoires », quelque fois d'usage obligatoire, qui servent à caractériser le rapport entre l'énonciateur et le contenu de l'énonciation, distinguant par exemple une information qui porte sur un fait dont l'énonciateur fut témoin, d'un autre fait dont il ne le fut point. Ce même rapport aurait été, à l'origine, exprimé par le vocable grec istorein, utilisé par Hérodote au sens de témoin et d'enquête : « le mot istoria [...] signifiant alors 'témoin oculaire' et, postérieurement, celui qui examine des témoins et obtient la vérité à travers l'enquête " (Arendt 1972, p. 69). Thucydide introduit L'Histoire de la Guerre du Péloponmèse en aflirmant, à la troisième personne, sa relation avec les faits : "Thucydide d'Athènes a écrit la guerre des Péloponnésiens et des Athéniens, comment ils l'ont faite les uns contre les autres. Il commence le récit de la guerre dès qu'elle éclate, ayant pronostiqué qu'elle allait prendre de grandes proportions et qu'elle serait plus digne de mention que celles qui avaient déjà eu lieu [...] » $(1999$, p. 3).

18. Ma définition s'approche de la thèse de F. Châtelet (1962) sur la naissance de l'histoire (et de la conscience historique) en Grèce entre les vil et $\mathrm{v}^{\mathrm{e}}$ siècles avant J.-C. Le rapprochement est intentionnel. Ce modèle rencontre une résonance dans l'ethnologie sud-américaine, spécialement dans les travaux de Turner sur le surgissement de la conscience historique chez les Kayapó (voir 1998a, 1993 ; pour la discussion de Turner sur les notions grecques et hébraïques de l'histoire, voir 1988b).

19. Pensons, entre autres exemples, aux santidades qui ont éclos sur la côte brésilienne durant le $\mathrm{XvI}^{\mathrm{e}}$ siècle (Vainfas 1995), à l'expulsion des Espagnols au XviI' ${ }^{\mathrm{e}}$ siècle par les Arawak sub-andins conduits par Juan Santos Atahualpa (Santos Granero 1993), aux mouvements millénaristes du Haut Rio Negro au XIXe siècle (Hill \& Wright 1988) ou encore aux événements plus récents, comme ce qui s'est passé chez les Canela dans les années soixante (Carneiro da Cunha 1986).

\section{RÉFÉRENCES BIBLIOGRAPHIQUES}

AnChieta, José de, [1554-1594] 1988. - Cartas : Informações, Fragmentos Históricos e Sermões (1554-1594), Belo Horizonte, Itatiaia/EDusP.

Arendt, Hannah, 1972. - Entre o Passado e o Futuro, São Paulo, Editora Perspectiva.

Carneiro da Cunha, Manuela, 1986 [1973]. - « Lógica do Mito e da Ação. O Movimento Messiânico Canela de 1963 ", in : Antropologia do Brasil. Mito, História, Etnicidade, São Paulo, Brasiliense/EDUSP, pp. 13-52.

ChÂtelet, François, 1962. - La Naissance de l'Histoire, Paris, Éditions de Minuit.

Chernel. Judith, 1988. - « Righting History in the Northwest Amazon : Myth, Structure, and History in an Arapaço Narrative ", in : J. D. Hill (ed.), Rethinking History and Myth: Indigenous South American Perspectives on the Past, Urbana, University of Illinois Press, pp. 35-49.

DaMatta, Roberto, 1970. — « Mito e Antimito entre os Timbira », in : C. Lévi-Strauss et al., Mito e Linguagem Social. Ensaios de Antropologia Estrutural, Rio de Janeiro, Tempo Brasileiro, pp. 77-106.

Évreux, Yves d', [1613] 1985. - Voyage au Nord du Brésil : fait en 1613 et 1614, Paris, Payot. 
Fausto, Carlos, 1999. - « Of Enemies and Pets : Warfare and Shamanism in Amazonia », American Ethnologist, 26 (4), pp. 933-956.

—, 2001a. - Inimigos Fiéis : História, Guerra e Xamanismo na Amazônia, São Paulo, EdusP.

—, 2001b. — «Si Dieu était Jaguar : cannibalisme et christianisme chez les Guarani », conférence à l'EPHE, Paris.

—, 2002. — «The Bones Affair : Indigenous Knowledge Practices in Contact Situations Seen from an Amazonian Case ", Journal of the Royal Anthropological Institute, 8 (4).

Gallors, Dominique T., 1994. - Mairi Revisitada. A Reintegração da Fortaleza de Macapá na Tradição Oral dos Waiãpi, Sào Paulo, NHu/USP - FAPESP.

Grenand, Françoise, 1982. - Et l'Homme devint Jaguar, Paris, L'Harmattan.

Hemming, John, 1987. - Amazon Frontier : the Defeat of the Brazilian Indians, London, MacMillan.

Heronotus, 1952. - The History of Herodotus, London, Enciclopaedia Britanica "Great Books of the Western World ».

HıLL, Jonathan D., 1988. — « Introduction : Myth and History », in : J. D. Hill (ed.), Rethinking History [...], op. cit.

Hill, Jonathan D. \& Robin Wright, 1988. - « Time, Narrative, and Ritual : Historical Interpretations from an Amazonian Society ", in : J. D. Hill (ed.), Rethinking History [...], op. cit., pp. 78-105.

IRELAND, Émilienne, 1988. - «Cerebral Savage : the Whiteman as Symbol of Cleverness and Savagery in Waurá Myth », in : J. D. Hill (ed.), Rethinking History [... ], op. cit., pp. 157-173.

Lévi-Strauss, Claude, 1971. - L'Homme mu, Paris, Plon.

—, 1991. - Histoire de lynx, Paris, Plon.

Melattı, Julio Cezar, 1967. - Índios e Criadores : a Situação dos Krahó na Área Pastoril do Tocantins, Rio de Janeiro, Instituto de Ciências Sociais da UFRJ.

Müller, Regina, 1990. - Os Asurini do Xingu : História e Arte, Campinas, SP, Editora da Unicamp.

Nimuendauú, Curt U., 1946. - The Eastern Timbira, Berkeley, University of California Press. —, 1956. - « Os Apinajé », Boletim do Museu Paraense Emilio Goeldi, Tomo XII.

Santos Granero, Fernando, 1993. — « Templos e Ferrarias : Utopia e Reinvenção Cultural no Oriente Peruano ", in : E. Viveiros de Castro \& M. Carneiro da Cunha (eds.), Amazônia : Etnologia e História Indigena, São Paulo, NHI/FAPESP, pp. 67-94.

Thevet, André, [1575] 1953. - « La Cosmographie Universelle », in : S. Lusagnet (ed.), Le Brésil et les Brésiliens : les Français en Amérique pendant la deuxième moitié du $\mathrm{xvI}^{\mathrm{e}}$ siècle, Paris, PUF.

TucíbIDEs, 1999. - História da Guerra do Peloponeso, Rio de Janeiro, Martins Fontes.

Turner, Terence, 1988a. - " History, Myth, and Social Consciousness among the Kayapó of Central Brazil », in : J. D. Hill (ed.), Rethinking History [...], op. cit., pp. 195-213.

—, 1988b. — « Commentary : Ethno-Ethnohistory : Myth and History in Native South American Representations of Contact », in : J. D. Hill (ed.) Rethinking History [... ], op. cit., pp. 235-281.

—, 1993. — « Da Cosmologia à História : Resistência, Adaptação e Consciência Social entre os Kayapó », in : E. Viveiros de Castro \& M. Carneiro da Cunha (eds.), Amazônia [...] ], op. cit., pp. 43-66. 
VAinfas, Ronaldo, 1995. - A Heresia dos Índios : Catolicismo e Rebeldia no Brasil Colonial, Sào Paulo, Companhia das Letras.

VIDAL, Lux, 1977. - Morte e Vida de uma Sociedade Indigena Brasileira, São Paulo, Hucitec/EDUSP.

Vieira, Antonio, [1654] 1943. - « Carta ao Pe. Francisco Gonçalves, Provincial do Brasil », in : S. Leite, História da Companhia de Jesus, vol. 3, Rio de Janeiro, Imprensa Nacional, pp. 316-337.

Viveiros de Castro, Eduardo, 1996. - «Os Pronomes Cosmológicos e o Perspectivismo Ameríndio », Mana : Estudos de Antropologia Social, 2 (2), pp. 115-144.

Wagner, Roy, 1981. - The Invention of Culture, Chicago, Chicago University Press. 